TR ANSACTIONS OF THE

AMERICAN MATHEMATICAL SOCIETY

Volume 181, July 1973

\title{
DIFFERENTIAL GEOMETRIC STRUCTURES ON PRINCIPAL TOROIDAL BUNDLES
}

BY

\author{
DAVID E. BLAIR, GERALD D. LUDDEN AND KENTARO YANO
}

ABSTRACT. Under an assumption of regularity a manifold with an f-structure satisfying certain conditions analogous to those of a Kähler structure admits a fibration as a principal toroidal buindle over a Kähler manifold. In some natural special cases, additional information about the bundle space is obtained. Finally, curvature relations between the bundle space and the base space are studied.

Let $M^{2 n+s}$ be a $C^{\infty}$ manifold of dimension $2 n+s$. If the structural group of $M^{2 n+s}$ is reducible to $U(n) \times O(s)$, then $M^{2 n+s}$ is said to have an f-structure of rank $2 n$. If there exists a set of 1 -forms $\left\{\eta^{1}, \cdots, \eta^{s}\right\}$ satisfying certain properties described in $\$ 1$, then $M^{2 n+s}$ is said to have an $f$-structure with complemented frames. In [1] it was shown that a principal toroidal bundle over a Kähler manifold with a certain connection has an $f$-structure with complemented frames and $d \eta^{1}=\cdots=d \eta^{s}$ as the fundamental 2-form. On the other hand, the following theorem is proved in $\$ 2$ of this paper.

Theorem 1. Let $M^{2 n+s}$ be a compact connected manifold with a regular normal f-structure. Then $M^{2 n+s}$ is the bundle space of a principal toroidal bundle over a complex manifold $N^{2 n}\left(=M^{2 n+s} / M\right)$. Moreover, if $M^{2 n+s}$ is a K-manifold, then $N^{2 n}$ is a Käbler manifold.

After developing a theory of submersions in $\$ 3$, we discuss in $\$ 4$ further properties of this fibration in the cases where $d \eta^{x}=0, x=1, \cdots, s$ and $d \eta^{x}=$ $a^{x} F, F$ being the fundamental 2-form of the $f$-structure. $N^{2 n}$

Finally in $\$ 5$ we study the relation between the curvature of $M^{2 n+s}$ and

Since $U(n) \times O(s) \subset O(2 n+s), M^{2 n+s}$ is a new example of a space in the class provided by Chern in his generalization of Kähler geometry [4]. S. I. Goldberg's paper [5] also suggests the study of framed manifolds as bundle spaces over Kähler manifolds with parallelisable fibers.

1. Normal f-structures. Let $M^{2 n+s}$ be a $2 n+s$-dimensional manifold with an $f$-structure. Then there is a tensor field $f$ of type $(1,1)$ on $M^{2 n+s}$ that is of rank

Received by the editors January 10, 1972 and, in revised form, April 18, 1972.

AMS (MOS) subject classifications (1969). Primary 5372; Secondary 5730, 5380.

Key words and phrases. Principal toroidal bundles, f-structures, Kähler manifolds. 
$2 n$ everywhere and satisfies

$$
f^{3}+f=0
$$

If there exist vector fields $\xi_{x}, x=1, \ldots, s$ on $M^{2 n+s}$ such that

$$
f \xi_{x}=0, \quad \eta^{x}\left(\xi_{y}\right)=\delta_{y}^{x}, \quad \eta^{x} \circ f=0, \quad f^{2}=-I+\eta^{y} \otimes \xi_{y},
$$

we say $M^{2 n+s}$ has an $f$-structure with complemented frames. Further we say that the $f$-structure is normal if

$$
[f, f]+d \eta^{x} \otimes \xi_{x}=0
$$

where $[f, f]$ is the Nijenhuis torsion of $f$. It is a consequence of normality that $\left[\xi_{x}, \xi_{y}\right]=0$. Moreover it is known that there exists a Riemannian metric $g$ on $M^{2 n+s}$ satisfying

$$
g(X, Y)=g(f X, f Y)+\sum_{x} \eta_{x}(X) \eta_{x}(Y)
$$

where $X$ and $Y$ are arbitrary vector fields on $M^{2 n+s}$. Define a 2 -form $F$ on $M^{2 n+s}$ by

$$
F(X, Y)=g(X, f Y)
$$

A normal $f$-structure for which $F$ is closed will be called a $K$-structure and a $K$-structure for which there exist functions $\alpha^{1}, \ldots, a^{s}$ such that $\alpha^{x} F=d \eta^{x}$ for $x=1, \ldots, s$ will be called an $S$-structure.

Lemma 1. If $M^{2 n+s}, n>1$, has an $S$-structure, then the $\alpha^{x}$ are all constant.

Proof. $\alpha^{x} F=d \eta^{x}$ so that $d \alpha^{x} \wedge F=0$ since $d F=0$. However $F \neq 0$ so $d \alpha^{x}=0$ and hence $\alpha^{x}$ is constant.

The special case where the $\alpha^{x}$ are all 0 or all 1 has been studied in [1]. Also, the following were proved.

Lemma 2. If $M^{<n+s}$ has a K-structure, the $\xi_{x}$ are Killing vector fields and $d \eta^{x}(X, Y)=-2\left(\widetilde{\nabla}_{Y} \eta^{x}\right)(X)$. Here $\tilde{\nabla}$ is the Riemannian connection of $g$ on $M^{2 n+s}$.

From Lemma 2, we can see that in the case of an $S$-structure $a^{x} f Y=$ $-2 \widetilde{\nabla}_{Y} \xi_{x}$.

Lemma 3. If $M^{2 n+s}$ bas a K-structure, then

$$
\left(\widetilde{\nabla}_{X} F\right)(Y, Z)=\frac{1}{2} \sum_{x}\left(\eta^{x}(Y) d \eta^{x}(f Z, X)+\eta^{x}(Z) d \eta^{x}(X, f Y)\right) .
$$

2. Proof of Theorem 1. In Chapter 1 of [9] R. S. Palais discusses quotient manifolds defined by foliations. In particular, a cubical coordinate system $\left\{U,\left(u^{1}, \ldots, u^{n}\right)\right\}$ on an $n$-dimensional manifold is said to be regular with respect 
to an involutive $m$-dimensional distribution if $\left\{\partial(m) / \partial u^{x}\right\}, x=1, \ldots, m$, is a basis of $M_{m}$ for every $m \in U$ and if each leat of $M$ intersects $U$ in at most one $m$-dimensional slice of $\left\{U,\left(u^{1}, \ldots, u^{n}\right)\right\}$. We say $\mathbb{M}$ is regular if every leaf of $M$ intersects the domain of a cubical coordinate system which is regular with respect to $M$.

In [9] it is proven that if $M$ is regular on a compact connected manifold $M$, then every leaf of $M$ is compact and that the quotient $M / M$ is a compact differentiable manifold. Moreover the leaves of $M$ are the fibers of a $C^{\infty}$ fibering of $M$ with base manifold $M / M$ and the leaves are all $C^{\infty}$ isomorphic.

We now note that the distribution $M$ spanned by the vector fields $\xi_{1}, \ldots, \xi_{s}$ of a normal $f$-structure is involutive. In fact we have by normality

$$
0=[f, f]\left(\xi_{y}, \xi_{z}\right)+d \eta^{x}\left(\xi_{y}, \xi_{z}\right) \xi_{x}=f^{2}\left[\xi_{y}, \xi_{z}\right]-\eta^{x}\left(\left[\xi_{y}, \xi_{z}\right]\right) \xi_{x}=-\left[\xi_{y}, \xi_{z}\right]
$$

from which it easily follows that $M$ is involutive. If $M$ is regular and the vector fields $\xi_{x}$ are regular we say that the normal $f$-structure is regular. Thus from the results of [9] we see that if $M^{2 n+s}$ is compact and has a regular normal $f$-structure, then $M^{2 n+s}$ admits a $C^{\infty}$ fibering over the $(2 n)$-dimensional manifold $N^{2 n}=$ $M^{2 n+s} / M$ with compact, $C^{\infty}$ isomorphic, fibers.

Since the distribution $M$ of a regular normal $f$-structure consists of $s$ 1-dimensional regular distributions each given by one of the $\xi_{x}$ 's, if $M^{2 n+s}$ is compact, the integral curves of $\xi_{x}$ are closed and hence homeomorphic to circles $S^{1}$. The $\xi_{x}$ 's being independent and regular show that the fibers determined by the distribution $M$ are homeomorphic to tori $T^{s}$.

Now define the period function $\lambda_{X}$ of a regular closed vector field $X$ by

$$
\lambda_{X}(m)=\inf \{t>0 \mid(\exp t X)(m)=m\} .
$$

For brevity we denote $\lambda_{\xi_{x}}$ by $\lambda_{x}$.W. M. Boothby and H. C. Wang [3] proved that $\lambda_{x}(m)$ is a differentiable function on $M^{2 n+s}$. We now prove the following

Lemma 4. The functions $\lambda_{x}$ are constants.

The proof of the lemma makes use of the following theorem of A. Morimoto [7].

Theorem (Morimoto [7]). Let $M$ be a complex manifold with almost complex structure tensor $J$. Let $X$ be an analytic vector field on $M$ such that $X$ and $J X$ are closed regular vector fields. Set $p(m)=\lambda_{X}(m)+\sqrt{-1} \lambda_{J X}(m)$. Then $p$ is a bolomorpbic function on $M$.

Proof of lemma. For $s$ even,

$$
\tilde{f}=f+\sum_{i=1}^{s / 2}\left(\eta^{i} \otimes \xi_{i}-\eta^{i^{*}} \otimes \xi_{i}\right), \quad i=1, \cdots, s / 2, i^{*}=i+s / 2,
$$


defines a complex structure on $M=M^{2 n+s}$ (cf. [6]). It is clear from the normality that $\xi_{x}$ is a holomorphic vector field. For $s$ odd, a normal almost contract structure $\left(\widetilde{f}, \xi_{0}, \eta_{0}\right)$ is defined where $\xi_{0}$ and $\eta_{0}$ generically denote one of the $\xi_{x}$ 's and $\eta_{x}$ 's respectively [6]. It is well known that this structure induces a complex structure $J$ on $M=M^{2 n+s} \times S^{1}$. Moreover, by the normality, $\xi_{0}$ considered as a vector field on $M$ is analytic. Then $p(m)=\lambda_{x}(m)+\sqrt{-1} \lambda_{x}(m)$ or $p((m, q))=$ $\lambda_{\xi_{0}}((m, q))+\sqrt{-1} \lambda_{J \xi_{0}}((m, q)), q \in S^{1}$, for $s$ odd, is a holomorphic function on $M$ by the theorem of Morimoto. Since $M$ is compact, $p$ must be constant. Thus $\lambda_{\dot{x}}$ is constant on $M$ and since $\lambda_{x}((m, q))=\lambda_{x}(m), \lambda_{x}$ is constant on $M^{2 n+s}$.

Let $C_{x}=\lambda_{x}(m)$, then the circle group $S_{x}^{1}$ of real numbers modulo $C_{x}$ acts on $M^{2 n+s}$ by $(t, m) \rightarrow\left(\exp t \xi_{x}\right)(m), t \in R$. Now the only element in $T^{s}=S_{1}^{1} \times \cdots$ $\times S_{s}^{1}$ with a fixed point in $M^{2 n+s}$ is the identity and since $M^{2 n+s}$ is a fiber space over $N^{2 n}$, we need only show that $M^{2 n+s}$ is locally trivial [3]. Let $\left\{U_{\alpha}\right\}$ be a cover of $N^{2 n}$ such that each $U_{a}$ is the projection of a regular neighborhood on $M^{2 n+s}$ and let $s_{\alpha}: U_{\alpha} \rightarrow M^{2 n+s}$ be the section corresponding to $u^{1}=$ constant, $\cdots, u^{s}=$ constant. Then the maps $\Psi_{\alpha}: U_{\alpha} \times T^{s} \rightarrow M^{2 n+s}$ defined by

$$
\Psi_{\alpha}\left(p, t_{1}, \cdots, t_{s}\right)=\left(\exp \left(t_{1} \xi_{1}+\cdots+t_{s} \xi_{s}\right)\right)\left(s_{\alpha}(p)\right)
$$

give coordinate maps for $M^{2 n+s}$.

Finally (cf. [1]) we note that $\gamma=\left(\eta^{1}, \ldots, \eta^{s}\right)$ defines a Lie algebra valued connection form on $M^{2 n+s}$ and we denote by $\tilde{\pi}$ the horizontal lift with respect to $\gamma$. Define a tensor field $J$ of type $(1,1)$ on $N^{2 n}$ by $J X=\pi_{*} f \pi x$. Then, since the distribution $\mathcal{Q}$ complementary to $\mathbb{N}$ is horizontal with respect to $\gamma$,

$$
J^{2} X=\pi_{*} f \tilde{\pi} \pi_{*} f \tilde{\pi} X=\pi_{*} f^{2} \tilde{\pi} X=-X .
$$

Moreover

$$
\begin{aligned}
& {[J, J](X, Y)=-[\therefore, Y]+\left[\pi_{*} f \tilde{\pi} X, \pi_{*} f \tilde{\pi} Y\right]-\pi_{*} \tilde{j}\left[\pi_{*} f \tilde{\pi} X, Y\right]-\pi_{*} f \tilde{\pi}\left[X, \pi_{*} f \tilde{\pi} Y\right]} \\
& =-\pi_{*}[\tilde{\pi} X, \tilde{\pi} Y]+\pi_{*}[\tilde{\pi} X, \tilde{f} \tilde{Y} Y]-\pi_{*} f \tilde{\pi} \pi_{*}[\tilde{f} \tilde{\pi} X, \tilde{\pi} Y]-\pi_{*} f \tilde{\pi} \pi_{*}[\tilde{\pi} X, \tilde{f} Y] \\
& =\pi_{*}\left(f^{2}\left[\tilde{\pi} X, \tilde{\pi} Y^{\prime}\right]-\eta^{x}([\tilde{\pi} X, \tilde{\pi} Y]), \xi_{x}\right)+\pi_{*}\left[\tilde{\pi} X, \tilde{\pi} Y^{Y}\right]-\pi_{*} f[\tilde{\pi} X, \tilde{\pi} Y]-\pi_{*} f[\tilde{\pi} X, f \tilde{\pi} Y] \\
& =\pi_{*}\left([f, f](\tilde{\pi} X, \tilde{\pi} Y)+d \eta^{x}(\tilde{\pi} X, \tilde{\pi} Y) \xi_{x}\right) \\
& =0 \text {. }
\end{aligned}
$$

Thus we see that $N^{2 n}$ is a complex manifold.

We define an Hermitian metric $G$ on $N^{2 n}$ by $G(X, Y)=g(\tilde{\pi} X, \tilde{\pi} Y)$. Indeed

$$
\begin{aligned}
\tilde{G}(J X, J Y) & =g\left(\tilde{\pi} \pi_{*} f \tilde{\pi} X, \tilde{\pi} \pi_{*} f \tilde{\pi} Y\right)=g(\tilde{\pi} X, f \tilde{\pi} Y) \\
& =g(\tilde{\pi} X, \tilde{\pi} Y)-\sum \eta^{x}(\tilde{\pi} X) \eta^{x}(\tilde{\pi} Y)=G(X, Y) .
\end{aligned}
$$


Now define the fundamental 2-form $\Omega$ by $\Omega(X, Y)=G(X, J Y)$. Then for vector fields $\widetilde{X}, \tilde{Y}$ on $M^{2 n+s}$ we have

$$
\begin{aligned}
& \pi^{*} \Omega(\tilde{X}, \tilde{Y})=\Omega\left(\pi_{*} \tilde{X}, \pi_{*} \tilde{Y}\right)=G\left(\pi_{*} \tilde{X}, J \pi_{*} \tilde{Y}\right) \\
& \quad=g\left(\tilde{\pi} \pi_{*} \tilde{X}, \tilde{\pi} J \pi_{*} \tilde{Y}\right)=g\left(-f^{2} \tilde{X}, \tilde{\pi} \pi_{*} f \tilde{Y}\right)=g\left(-f^{2} \tilde{X}, f \tilde{Y}\right)=g(\tilde{X}, f \widetilde{Y})=F(\tilde{X}, \tilde{Y}) .
\end{aligned}
$$

Thus $F=\pi^{*} \Omega$. If now $d F=0$, then $0=d \pi^{*} \Omega=\pi^{*} d \Omega$ and hence $d \Omega=0$ since $\pi^{*}$ is injective. Thus the manifold $N^{2 n}$ is Kählerian.

3. Submersions. Let $\widetilde{\nabla}$ denote the Riemannian connection of $g$ on $M^{2 n+s}$. Since the $\xi_{x}$ 's are Killing, $g$ is projectable to the metric $G$ on $N^{2 n}$. Then following [8] the horizontal part of $\tilde{\nabla}_{\pi} \tilde{\pi} Y$ is $\tilde{\pi}_{X} Y$ where as we shall see $\nabla$ is the Riemannian connection of $\underset{\sim}{G}$. Now for an $S$-structure we have seen that $\tilde{\nabla} \tilde{X}^{\xi} \xi_{x}=$ $a^{x} f \tilde{X}$ for any vector field $\tilde{X}$ on $M^{2 n+s}$. By normality $f$ is projectable $\left(\Omega_{\xi_{x}} f=0\right)$ and the $\alpha^{x}$ 's are constants; thus we can write

$$
\tilde{\nabla} \tilde{\pi}_{X} \xi_{x}=-\tilde{\pi} H_{x} X
$$

where $H_{x}$ is a tensor field of type $(1,1)$ on $N^{2 n}$.

We can now find the vertical part of $\tilde{\nabla} \tilde{\pi} x$.

$$
g\left(\tilde{\nabla}_{\pi} \tilde{\pi} Y, \xi_{x}\right)=-g\left(\tilde{\pi} Y, \tilde{\nabla}_{\tilde{\pi} X} \xi_{x}\right)=g\left(\tilde{\pi} Y, \tilde{\pi} H_{x} X\right) .
$$

Thus we can write

$$
\tilde{\nabla}_{\pi} \tilde{\pi} Y=\tilde{\pi} \nabla_{X} Y+b^{x}(X, Y) \xi_{x}
$$

where each $b^{x}$ is a tensor field of type $(0,2)$ and

$$
G\left(H_{x} X, Y\right)=h^{x}(X, Y)
$$

Lemma 5. $\Omega_{\xi_{x}}(\tilde{\pi} X)=0$ for any vector field $X$ on $N^{2 n}$, where $\Omega_{\xi_{x}}$ is the operator of Lie differentiation in the $\xi_{x}$ direction.

Proof. We have that $g\left(\xi_{y}, \tilde{\pi} X\right)=0$ for $y=1, \ldots, s$. By Lemma 2, the $\xi_{x}$ are Killing, that is $\Omega_{\xi_{x}} g=0$. From the normality of $f, \Omega_{\xi_{x}} \xi_{y}=0$. Hence, we have that

$$
g\left(\xi_{y}, 2_{\xi_{x}}(\tilde{\pi} Y)\right)=0, \quad y=1, \cdots, s,
$$

and so $\Omega_{\xi_{x}}(\tilde{\pi} X)$ is horizontal. However,

$$
\pi_{*} \Omega_{\xi_{x}}(\tilde{\pi} X)=\pi_{*}\left[\xi_{x}, \tilde{\pi} X^{\prime}\right]=\left[\pi_{*} \xi_{x}, \pi_{*} \tilde{\pi} X\right]=0
$$

and so $\Omega_{\xi_{x}}(\tilde{\pi} X)$ is vertical. 
Using the lemma we see that $\widetilde{\nabla}_{\xi_{x}} \tilde{\pi} X=\widetilde{\nabla} \tilde{\pi} X_{x}$ for any vector field $X$ on $N^{2 n}$. Since $\xi_{x}$ is Killing, we have

$$
0=g\left(\tilde{\nabla}_{\tilde{\pi} X} \xi_{x}, \tilde{\pi} X\right)=-g\left(\xi_{x}, \tilde{\nabla} \tilde{\pi} X \tilde{\pi} X\right)=-g\left(\xi_{x}, b^{y}(X, X) \xi_{y}\right)=-b^{x}(X, X)
$$

for all $X$. That is to say $b^{x}(X, Y)=-b^{x}(Y, X)$ for all $X$ and $Y$. Now we have that

$$
\begin{aligned}
0 & =\tilde{\nabla}_{\pi}(\tilde{\pi} Y)-\tilde{\nabla}_{\tilde{\pi} Y}(\tilde{\pi} X)-[\tilde{\pi} X, \tilde{\pi} Y] \\
& =\tilde{\pi}\left(\nabla_{X} Y-\nabla_{Y} X-[X, Y]\right)+\left(b^{x}(X, Y)-b^{x}(Y, X)+d \eta^{x}(\tilde{\pi} X, \tilde{\pi} Y)\right) \xi_{x} \\
& =\tilde{\pi}\left(\nabla_{X} Y-\nabla_{Y} X-[X, Y]\right)+\left(2 b^{x}(X, Y)+d \eta^{x}(\tilde{\pi} X, \tilde{\pi} Y)\right) \xi_{x},
\end{aligned}
$$

where we have used the following lemma.

Lemma 6. $[\tilde{\pi} X, \tilde{\pi} Y]=\tilde{\pi}[X, Y]-d \eta^{x}(\tilde{\pi} X, \tilde{\pi} Y) \xi_{x}$.

Proof. Since $\pi_{*}[\tilde{\pi} X, \tilde{\pi} Y]=\left[\pi_{*} \tilde{\pi} X, \pi_{*} \tilde{\pi} Y\right]=[X, Y]$ we see that $\tilde{\pi}[X, Y]$ is the horizontal part of $[\tilde{\pi} X, \tilde{\pi} Y]$. By Lemma 2, we have

$$
d \eta^{x}(\tilde{\pi} X, \tilde{\pi} Y)=-2\left(\tilde{\nabla}_{\tilde{\pi} Y} \eta^{x}\right)(\tilde{\pi} X)=-2 g\left(\tilde{\nabla}_{\tilde{\pi} Y} \xi_{x}, \tilde{\pi} X\right)=+2 g\left(\xi_{x}, \tilde{\nabla}_{\tilde{\pi} Y} \tilde{\pi} X\right) .
$$

Also $d \eta^{x}(\tilde{\pi} X, \tilde{\pi} Y)=-d \eta^{x}(\tilde{\pi} Y, \tilde{\pi} X)=-2 g\left(\xi_{x}, \tilde{\nabla} \tilde{\pi} X \tilde{\pi} Y\right)$. Thus

$$
2 d \eta^{x}(\tilde{\pi} X, \tilde{\pi} Y)=2 g\left(\xi_{x}, \tilde{\nabla}_{\tilde{\pi} Y} \tilde{\pi} X-\tilde{\nabla}_{\tilde{\pi} X} \tilde{\pi} Y\right)
$$

or

$$
d \eta^{x}(\tilde{\pi} X, \tilde{\pi} Y) \xi_{x}=\sum_{x} g\left(\xi_{x},[\tilde{\pi} X, \tilde{\pi} Y]\right) \xi_{x}=\text { vertical part of }[\tilde{\pi} X, \tilde{\pi} Y]
$$

From (6) we see $\nabla_{X} Y-\nabla_{Y} X-[X, Y]=0$ and $b^{x}(X, Y)=-1 / 2 d \eta^{x}(\tilde{\pi} X, \tilde{\pi} Y)$. Furthermore,

$$
\begin{aligned}
X G(Y, Z) & =\tilde{\pi} X g(\tilde{\pi} Y, \tilde{\pi} Z)=g\left(\tilde{\nabla}_{\tilde{\pi} X} \tilde{\pi} Y, \tilde{\pi} Z\right)+g\left(\tilde{\pi} Y, \tilde{\nabla}_{\tilde{\pi} X} \tilde{\pi} Z\right) \\
& =g\left(\tilde{\pi} \nabla_{X} Y, \tilde{\pi} Z\right)+g\left(\tilde{\pi} Y, \tilde{\pi} \nabla_{X} Z\right)=G\left(\nabla_{X} Y, Z\right)+G\left(Y, \nabla_{X} Z\right) .
\end{aligned}
$$

Thus, we have the following proposition.

Proposition. $\nabla$ is the Riemannian connection of $G$ on $N^{2 n}$.

4. The $S$-structure case. Let $M^{2 n+s}, n>1$, be a manifold with an $S$-structure. Then, as we have seen, there exist constants $\alpha^{x}, x=1, \ldots, s$, such that $\alpha^{x} F=$ $d \eta^{x}$. We will consider two cases, namely $\Sigma_{x}\left(a^{x}\right)^{2}=0$ and $\Sigma_{x}\left(a^{x}\right)^{2} \neq 0$.

In the first case each $d \eta_{x}=0$ and by Lemma 2 each $\xi_{x}$ is Killing, hence the 
regular vector fields $\xi_{1}, \ldots, \xi_{s}$ are parallel on $M^{2 n+s}$. Moreover the complementary distribution $\mathcal{Q}$ (projection map is $-f^{2}=I-\eta^{x} \otimes \xi_{x}$ ) is parallel. If now the distribution $\mathfrak{Q}$ is also regular, we have a second fibration of $M^{2 n+s}$ with fibers the integral submanifolds $L^{2 n}$ of $\mathscr{L}$ and base space an $s$-dimensional manifold $N^{s}$. Thus by a result of A. G. Walker [10] we see that although $M^{2 n+s}$ is not necessarily reducible (even though it is locally the product of $N^{2 n}$ and $T^{s}$ ) it is a covering space of $N^{2 n} \times N^{s}$ and is covered by $L^{2 n} \times T^{s}$. In summary we have

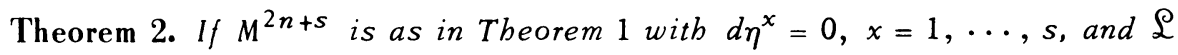
regular, then $M^{2 n+s}$ is a covering space of $N^{2 n} \times N^{s}$, where $N^{s}$ is the base space of the fibration determined by $\mathcal{L}$.

Now as in Theorem 1 , since the $\xi_{x}{ }^{\prime} s, x=1, \cdots, s$, are regular, we could fibrate by any $s-t$ of them to obtain a fibration of $M^{2 n+s}$ as a principal $T^{s-t}$ bundle over a manifold $P^{2 n+t}$. By normality the remaining $t$ vector fields are projectable to $P^{2 n+t}$. Moreover they are regular on $P^{2 n+t}$; for if not, the ir integral curves would be dense in a neighborhood $U$ over which $M^{2 n+s}$ is trivial with compact fiber $T^{s-t}$ contradicting their regularity on $M^{2 n+s}$. Thus $P^{2 n+t}$ is a principal $T^{t}$ bundle over $N^{2 n}$.

Theorem 3. If $M^{2 n+s}, n>1$, is as in Theorem 1 with $d \eta^{x}=\alpha^{x} F$ and $\Sigma_{x}\left(a^{x}\right)^{2} \neq 0$, then $M^{2 n+s}$ is a principal $T^{s-1}$ bundle over a principal circle bundle $P^{2 n+1}$ over $N^{2 n}$ and the induced structure on $P^{2 n+1}$ is a normal contact metric (Sasakian) structure.

Proof. Without loss of generality we suppose $\alpha^{s} \neq 0$. Then fibrating as above by $\xi_{1}, \ldots, \xi_{s-1}$ we have that $M^{2 n+s}$ is a principal $T^{s-1}$ bundle over a principal circle bundle $P^{2 n+1}$ over $N^{2 n}$. Let $p: M^{2 n+s} \rightarrow P^{2 n+1}$ denote the projection map. By normality $f, \xi_{s}, \eta^{s}$ are projectable, so we define $\phi, \xi, \eta$ on $P^{2 n+1}$ by

$$
\phi X=p_{*} f \tilde{p} X, \quad \xi=p_{*} \xi_{s}, \quad \eta(X)=\eta^{s}(\tilde{p} X)
$$

where $\tilde{p}$ denotes the horizontal lift with respect to the connection $\left(\eta^{1}, \cdots, \eta^{s-1}\right)$ considered as a Lie algebra valued connection form as in the proof of Theorem 1. Then by a straight-forward computation we have

$$
\eta(\xi)=1, \quad \phi \xi=0, \quad \eta \circ \phi=0, \quad \phi^{2}=-I+\xi \otimes \eta, \quad[\phi, \phi]+\xi \otimes d \eta=0,
$$

that is, $(\phi, \xi, \eta)$ is a normal almost contact structure on $P^{2 n+1}$. Defining a metric $\dot{g}$ by $\dot{g}(X, Y)=g(\tilde{p} X, \tilde{p} Y)$ we have $\dot{g}(X, \xi)=\eta(X)$ and $\dot{g}(\phi X, \phi Y)=\dot{g}(X, Y)$ $\eta(X) \eta(Y)$. Moreover setting $\Phi(X, Y)=\dot{g}(X, \phi Y)$ we obtain $F=p^{*} \Phi$. Thus since 


$$
\begin{aligned}
& d \eta^{s}=\alpha^{s} F, p^{*} \Phi=d \eta^{s} / \alpha^{s} \text { and } \\
& \begin{aligned}
\Phi(X, Y) & =g(\tilde{p} X, \tilde{p} \phi Y)=d \eta^{s}(\tilde{p} X, \tilde{p} Y) / \alpha^{s} \\
& =\left(X \eta(Y)-Y \eta(X)-\eta^{s}([\tilde{p} X, \tilde{p} Y])\right) / \alpha^{s}=d \eta(X, Y) / \alpha^{s}
\end{aligned}
\end{aligned}
$$

since $\eta^{s}$ is horizontal. Thus we have that $\eta_{\wedge}(d \eta)^{n}=\eta_{\wedge}\left(\alpha^{s} \Phi\right)^{n} \neq 0$ and hence that $P^{2 n+1}$ has a normal contact metric structure with $\xi$ regular.

Remark 1. While it is already clear that $P^{2 n+1}$ is a principal circle bundle over $N^{2 n}$, it now also follows from the well-known Boothby-Wang and Morimoto fibrations.

Remark 2. Under the hypotheses of Theorem 3, it is possible to assume without loss of generality that $\alpha^{x}$ equals 0 or $1 / \sqrt{ } t$ where $t$ is the number of nonzero $\alpha^{x}$ and hence there exist constants $\beta_{q}^{x}, q=1, \ldots, s-1$, such that $\bar{\eta}^{q}=$ $\Sigma_{x} \beta_{q}^{x} \eta^{x}$ and $\bar{\eta}^{s}=\Sigma_{x} \alpha^{x} \eta^{x}$ are 1 -forms with $d \bar{\eta}^{q}=0$ and $d \bar{\eta}^{s}=F$. Then $f, \bar{\eta}^{x}$ and the dual vector fields $\bar{\xi}_{x}$ again define a $K$-structure on $M^{2 n+s}$. If now this $K$-structure is regular, then, since the distribution spanned by $\bar{\xi}_{1}, \ldots, \bar{\xi}_{s-1}$ and its complement are parallel, $M^{2 n+s}$ is a covering of the product of $P^{2 n+1}$ and a manifold $P^{s-1}$ as in the proof of Theorem 2 .

Remark 3. In [1] one of the authors gave the following example of an $S$-manifold as a generalization of the Hopf-fibration of the odd-dimensional sphere over complex projective space, $\pi^{\prime}: S^{2 n+1} \rightarrow P C^{n}$. Let $\Delta$ denote the diagonal map and define a space $H^{2 n+s}$ by the diagram

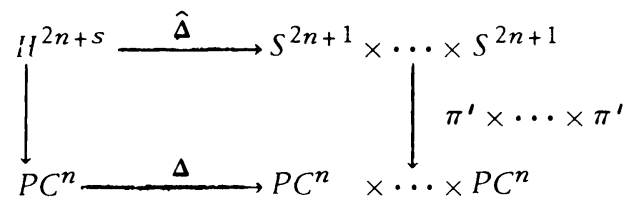

that is $H^{2 n+s}=\left\{\left(P_{1}, \cdots, P_{s}\right) \in S^{2 n+1} \times \cdots \times S^{2 n+1} \mid \pi^{\prime}\left(P_{1}\right)=\cdots=\pi^{\prime}\left(P_{s}\right)\right\}$ and thus $H^{2 n+s}$ is diffeomorphic to $S^{2 n+1} \times T^{s-1}$. Further properties of the space $H^{2 n+s}$ are given in [1], [2].

If however the $d \eta^{x}$ 's are independent then there can be no intermediate bundle $P^{2 n+t}$ over $N^{2 n}$ such that $M^{2 n+s}$ is trivial over $P^{2 n+t}$.

Remark 4. If $M^{2 n+s}$ is as in Theorem 1 with the $d \eta^{x}$ 's independent, then there is no fibration by $s-t$ of the $\xi_{x}$ 's yielding a principal toroidal bundle $P^{2 n+t}$ over $N^{2 n}$ such that $M^{2 n+s}=P^{2 n+t} \times T^{s-t}$. For suppose $P^{2 n+t}$ is such an intermediate bundle, then it is necessary that $\tilde{\nabla} \tilde{\pi} X \xi_{x}=0$ (see e.g. [8]) and thus the $\eta^{x}$ 's are parallel contradicting the independence of the $d \eta^{x}$ s.

5. Curvature. Let $\tilde{R}$ and $R$ denote the curvature tensors of $\tilde{\nabla}$ and $\nabla$ respectively. Then 


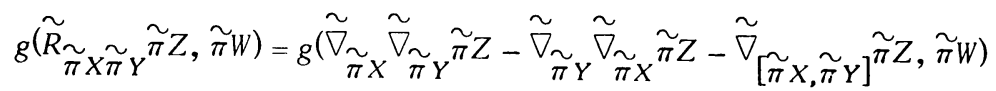

$$
\begin{aligned}
& =g\left(\tilde{\nabla}_{\pi}\left(\tilde{\pi} \nabla_{Y} Z+b^{x}(Y, Z) \xi_{x}\right)-\tilde{\nabla}_{\tilde{\pi} Y}\left(\tilde{\pi} \nabla_{X} Z+b^{x}(X, Z) \xi_{x}\right)\right. \\
& \left.-\tilde{\nabla} \tilde{\pi}[X, Y]-d \eta x(\tilde{\pi} X, \tilde{\pi} Y) \xi_{x} \tilde{\pi} Z, \tilde{\pi} W\right) \\
& =g\left(\tilde{\pi} \nabla_{X} \nabla_{Y} Z-b^{x}(Y, Z) \tilde{\pi}\left(H_{x} X\right)-\tilde{\pi} \nabla_{Y} \nabla_{X} Z+b^{x}(X, Z) \tilde{\pi}\left(H_{x} Y\right)\right.
\end{aligned}
$$

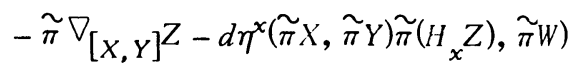

$$
\begin{aligned}
& =G\left(R_{X Y} Z, W\right)-\sum_{x}\left(b^{x}(Y, Z) b^{x}(X, W)-b^{x}(X, Z) b^{x}(Y, W)+d \eta^{x}(\tilde{\pi} X, \tilde{\pi} Y) b^{x}(Z, W)\right) \\
& \left.=G\left(R_{X Y} Z, W\right)-\sum_{x}\left(b^{x}(Y, Z) b^{x}(X, W)-i^{x}(X, Z) i\right)^{x}(Y, W)-2 b^{x}(X, Y) b^{x}(Z, W)\right) .
\end{aligned}
$$

In [1], one of the present authors developed a theory of manifolds with an $f-$ structure of constant $f$-sectional curvature. This is the analogue of a complex manifold of constant holomorphic curvature. A plane section of $M^{2 n+s}$ is called an $f$-section if there is a vector $X$ orthogonal to the distribution spanned by the $\xi_{x}$ 's such that $\{X, f X\}$ is an orthonormal pair spanning the section. The sectional curvature of this section is called an f-sectional curvature and is of course given by $g\left(\tilde{R}_{X f X} X, f X\right) \cdot M^{2 n+s}$ is said to be of constant $f$-sectional curvature if the $f$-sectional curvatures are constant for all $f$-sections. This is an absolute constant. We then have the following theorem.

Theorem 5. If $M^{2 n+s}$ is a compact, connected manifold with a regular S-structure of constant f-sectional curvature $c$, then $N^{2 n}$ is a Käbler manifold of constant bolomorphic curvature.

Proof. That $N^{2 n}$ is Kähler follows from Theorem 1. By definition there exist $\alpha^{1}, \cdots, a^{s}$, necessarily constant such that $\alpha^{x} F=d \eta^{x}$. If $X$ is a unit vector on $N^{2 n}$, then we have

$$
\begin{aligned}
G\left(R_{X J X} J X, X\right)= & g\left(\tilde{R} \tilde{\pi} X \tilde{\pi} J X^{\tilde{\pi} J} X, \tilde{\pi} X\right) \\
& +\sum_{x}\left(1 / 2 \alpha^{x} F(\tilde{\pi} J X, \tilde{\pi} J X) 1 / 2 \alpha^{x} F(\tilde{\pi} X, \tilde{\pi} X)\right. \\
& \quad-1 / 2 \alpha^{x} F(\tilde{\pi} X, \tilde{\pi} J X) 1 / 2 \alpha^{x} F(\tilde{\pi} J X, \tilde{\pi} X) \\
& \left.\quad-2(1 / 2) \alpha^{x} F(\tilde{\pi} X, \tilde{\pi} J X) 1 / 2 \alpha^{x} F(\tilde{\pi} J X, \tilde{\pi} X)\right) \\
= & c+3 / 4 \sum_{x}\left(\alpha^{x}\right)^{2}(F(\tilde{\pi} X, f \tilde{\pi} X))^{2} \\
= & c+3 / 4 \sum_{x}\left(\alpha^{x}\right)^{2}, \quad \text { which is constant. }
\end{aligned}
$$


Remark. This agrees with the results in [1] on $H^{2 n+s} \cdot H^{2 n+s}$ is a principal toroidal bundle over $P C^{n}$ and $P C^{n}$ is of constant holomorphic curvature equal to 1 . Also, $a^{x}=1$ for $x=1, \ldots, s$ and $H^{2 n+s}$ was found to be of constant $f$ sectional curvature equal to $1-3 s / 4$.

\section{REFERENCES}

1. D. E. Blair, Geometry of manifolds with structural group $\mathrm{U}(n) \times \mathcal{O}(s)$, J. Differential Geometry 4 (1970), 155-167. MR 42 \#2403.

2. - On a generalization of the Hopf fibration, An. Univ. "Al. I. Cuza" Iasi 17 (1971), 171-177.

3. W. M. Boothby and H. C. Wang, On contact manifolds, Ann. of Math. (2) 68 (1958), 721-734. MR $22 \# 3015$.

4. S. S. Chern, On a generalization of Kähler geometry, Algebraic Geometry and Topology (A Sympos. in Honor of S. Lefschetz), Princeton Univ. Press, Princeton, N. J., 1957, pp. 103-121. MR 19, 314.

5. S. I. Goldberg, A generalization of Kähler geometry, J. Differential Geometry 6 (1972), 343-355.

6. S. I. Goldberg and K. Yano, On normal globally framed f-manifolds, Tôhoku Math. J. 22 (1970), 362-370.

7. A. Morimoto, On normal almost contact structures with a regularity, Tôhoku Math. J. (2) 16 (1964), 90-104. MR 29 \#549.

8. B. O'Neill, The fundamental equations of a submersion, Michigan Math. J. 13 (1966), 459-469. MR $34 \# 751$.

9. R. S. Palais, A global formulation of the Lie theory of transformation groups, Mem. Amer. Math. Soc. No. 22 (1957). MR 22 \#12162.

10. A. G. Walker, The fibring of Riemannian manifolds, Proc. London Math. Soc. (3) 3 (1953), 1-19. MR 15, 159.

DEPARTMENT OF MATHEMATICS, MICHIGAN STATE UNIVERSITY, EAST LANSING, MICHIG AN 48823 